\title{
In search for effective and safe drugs against SARS-CoV-2: Part III] The electronic factors of Remdesivir and the naturally extracted Aspirochlorine drugs
}

\author{
M. S. A. Abdel-Mottaleb 1 and Yousra Abdel-Mottaleb2 \\ 1Department of Chemistry, Faculty of Science, Ain Shams University (ASU), Abbassia 11566, \\ Cairo, Egypt \\ 2Department of Pharmacology, Toxicology and Biochemistry, Faculty of Pharmaceutical \\ Sciences and Pharmaceutical Industries, Future University in Egypt (FUE), New Cairo 11835, \\ Egypt
}
Corresponding author: m.s.abdelmottaleb@sci.asu.edu.eg ihttps://orcid.org/0000-0002-4437-7040
2https://orcid.org/0000-0002-4327-4920

\begin{abstract}
:
An ongoing theme of the COVID-19 pandemic is the need for the widespread availability of efficient and safe drugs to treat infected people. The use of Remdesivir in COVID-19 has gotten emergency use authorization (EUA) from the US Food and Drug Administration (FDA) based on preliminary data. With limited treatment choices and high-risk side effects, a search for improved treatment is urgently needed. The two target proteins implicated in the pathogenesis of COVID19 are angiotensin-converting enzyme 2 (ACE2), and the specific peptide sequences of the coronavirus S-protein. In this frame, Remdesivir (an antiviral drug used for Ebola virus) and aspirochlorine (a natural product found in Aspergillus oryzae) were selected, and their binding to specific peptide sequences of the coronavirus S-protein: ACE2 interface-drug binding adduct were computed. The stable intermolecular adducts between the chosen drug molecules with the $\mathrm{S}$ protein and ACE2 result in limited host-virus interactions. Electronic characteristics such as the electrophilicity and nucleophilicity indices, based on the HOMO - LUMO frontier orbitals of the drug molecules, showed that both drugs act as electron sinks to shield ACE2 from interacting with the $\mathrm{S}$ protein. Aspirochlorine acts as an electron acceptor (electrophile) toward both individual targets, the ACE2, and S proteins (nucleophiles). Aspirochlorine electronically shields ACE2 from the interaction with $S$ proteins by sinking the electronic charge of the $S$ protein. The electrophilicity and nucleophilicity parameters of remdesivir are higher than those of ACE2, and both molecules
\end{abstract}


are bound via hydrogen bonding intermolecular interactions without electron transfer. Remdesivir also shields ACE2 from the S protein in its stable association. The results obtained strongly suggest the beneficial use of both drugs. Both drugs offer protection and/or treatment against the coronavirus S-protein COVID-19. However, the presence of remdesivir is very promising in the case of an already formed S protein - ACE2 adduct. In vivo and in vitro experimental validation of remdesivir is currently subjected to global underway testing.

Keywords: Simulations, Aspirochlorine, Remdesivir, Reactivity indices, Binding energy

\section{1- Introduction}

Remdesivir (Figure 1) is a prodrug that metabolizes into its active form GS-441524 (adenosine nucleoside analog), which interferes with the action of viral RNA-dependent RNA polymerase triggering decreased viral RNA multiplication.[1, 2] In the case of respiratory syncytial virus, the drug causes the RNA-dependent RNA polymerases to stop. Remdesivir is a broad-spectrum antiviral medication developed by the biopharmaceutical company Gilead Sciences [1]. As of 2020, remdesivir is under investigation as a specific treatment for COVID-19 and has been approved as EUA by the US FDA for treatment for those hospitalized with severe disease [3].

Aspirochlorine (Figure 2) is an unusual antifungal cyclopeptide produced by Aspergillus oryzae, an important mold used for food fermentation [4, 5]. This disulfide-containing compound (shown in Figure 2) has been found to display favorable cytotoxic properties towards the HL-60 cell line, which is a promyelocytic cell line derived from human leukemia [6]. 

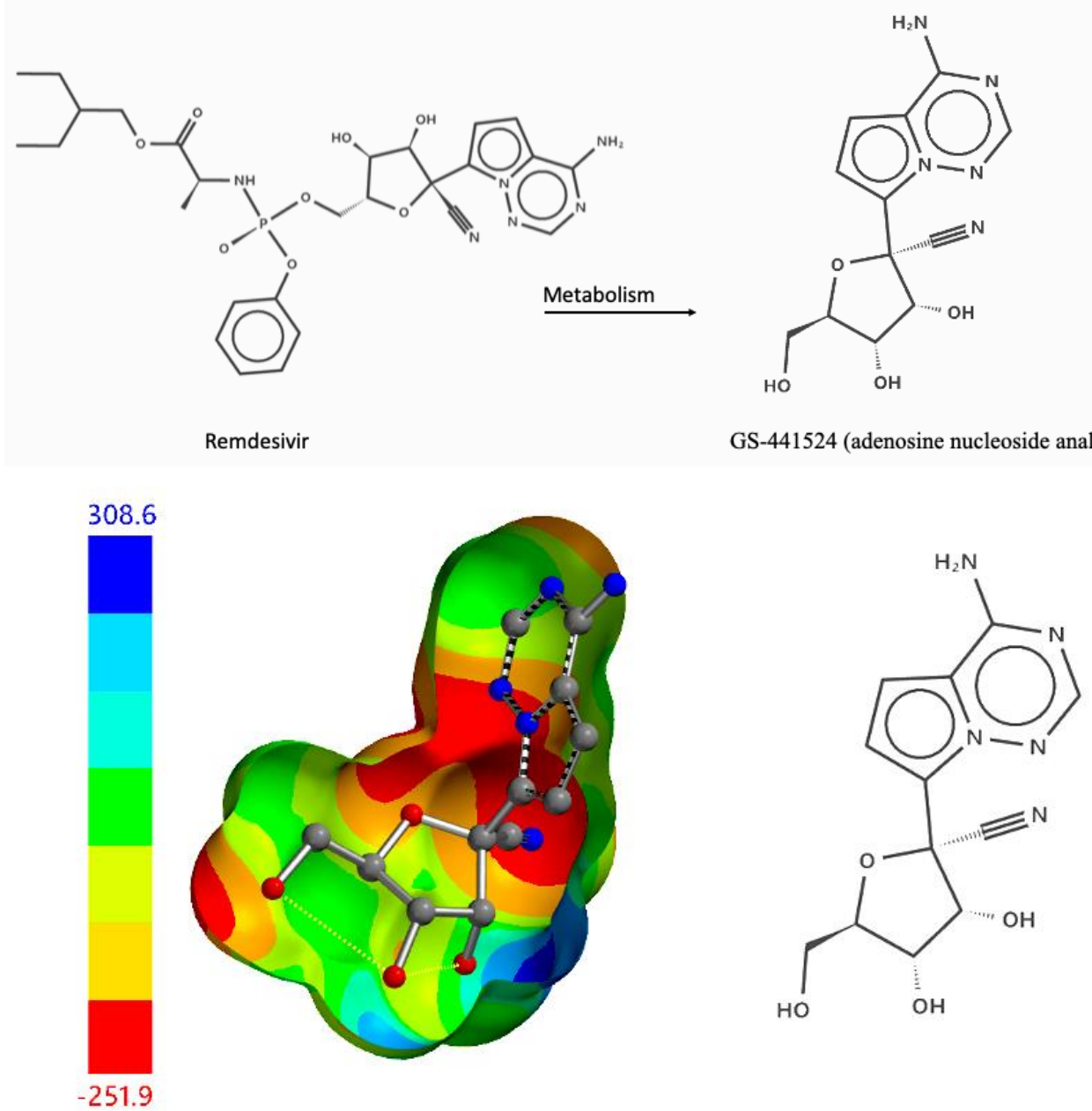

Figure 1. The metabolism of remdesivir to the reactive GS-441524 adenosine nucleoside analog (upper pane). ESP map (color code values are in $\mathrm{kJ}$ ) showing the electron rich (red color) and the electron poor (blue color) regions. 

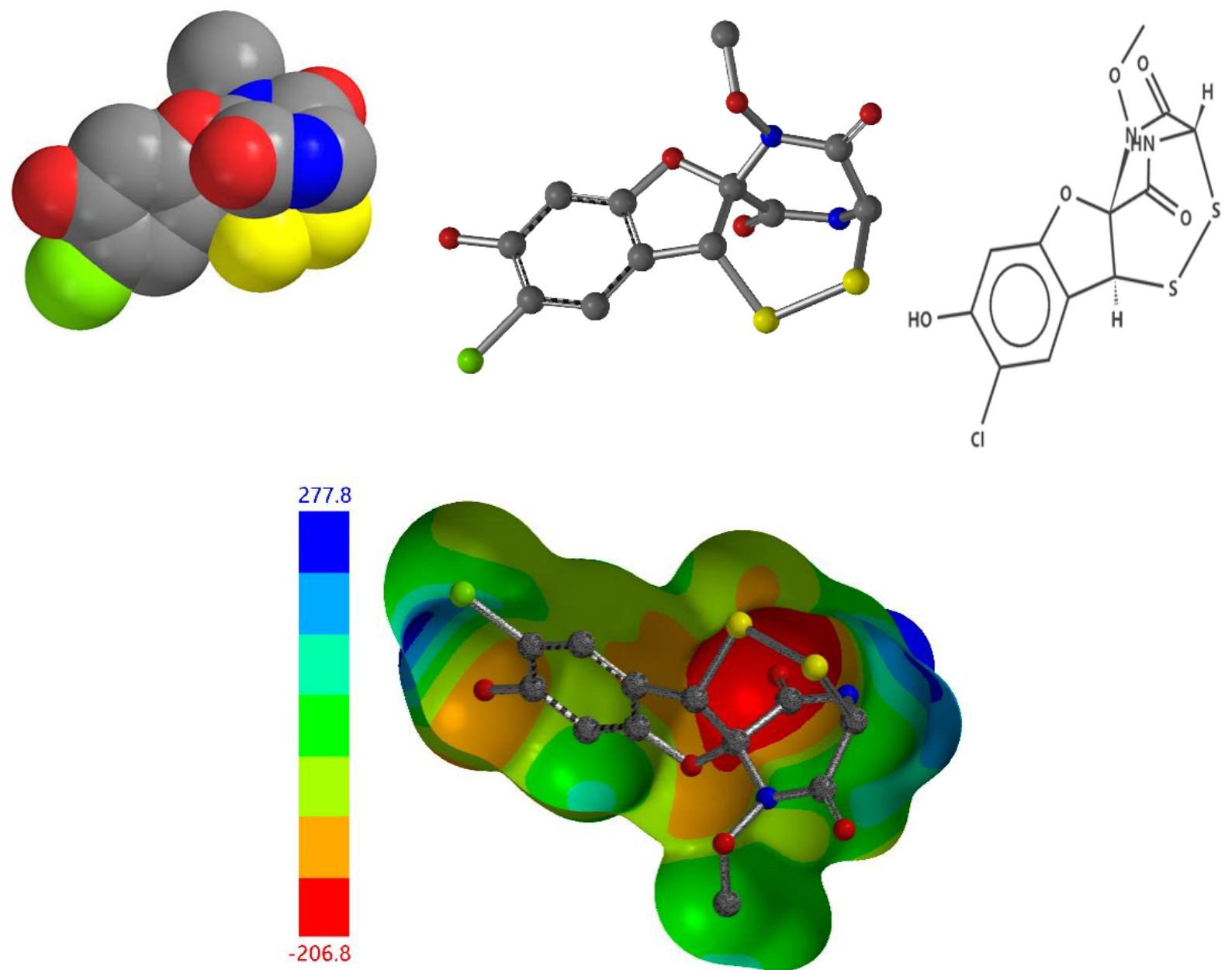

Figure 2. Aspirochlorine structure with different representation models. Space filling structure without the hydrogen atoms at the left side where grey, blue, red, yellow, and green colors represent carbon, nitrogen, oxygen, sulfur and chlorine atoms, respectively. The lower pane depicts ESP map of aspirochlorine.

Continuing our research on this timely issue of global interest [7,8], our goal is to predict whether remdesivir and aspirochlorine drugs will bind to a target (ACE2 or S protein) and, if so, how strongly. Simulations will be performed to estimate the strength of the intermolecular interaction between the drug molecule and its biological target (ACE2 and S protein). Computations will be also done to predict the reactivity parameters of the drug molecules and their electronic susceptibility to interactions with the biological target that may occur when the drug molecule binds to it. 


\section{2- Simulation Methodology}

The molecular mechanics method "Merck molecular force field (MMFF)", built in Spartan 18 parallel suite (core>16) package www.Wavefun.com, [9] was performed to achieve the equilibrium geometry of the interacting molecules. Single-point computations are then applied to the molecular mechanically optimized equilibrium geometry with DFT using the well-known longrange corrected hybrid functional wB97XD, which includes an empirical dispersion [10] method with a 6-31G(d) basis set. The methods used are quite accurate and non-expensive. The conductorlike polarizable continuum model (CPCM) was used for water solvation.[11]

\section{3- Results and Discussion}

Table 1 and Figures 3, 4, 5, and 6 summarize the numerical and graphical results obtained for the intermolecular interactions between the chosen drugs and ACE2 and S protein. In the present study, we followed the same procedure reported previously $[7,8]$ to compute the binding energy (BE) using the well-known equation:

BEadduct $=$ Eadduct $-(\Sigma$ Einteracting species $)$

Table 1. The energies of the frontier orbitals, the reactivity indices (in $\mathrm{eV}$ ) and the binding energy (in kcal/mol) of Remdesivir (R) and Aspirochlorine (A) drugs.

\begin{tabular}{|c|c|c|c|c|c|c|c|c|c|c|c|c|c|c|}
\hline \multirow{2}{*}{$\begin{array}{l}\text { Molecule/ } \\
\text { complex }\end{array}$} & \multicolumn{2}{|c|}{ HOMO } & \multicolumn{2}{|c|}{ LUMO } & \multicolumn{2}{|c|}{$\mu$} & \multicolumn{2}{|c|}{$\eta$} & \multicolumn{2}{|c|}{$\omega$} & \multicolumn{2}{|c|}{$\mathbf{N}$} & \multicolumn{2}{|c|}{ BE } \\
\hline & $\mathrm{R}$ & A & $\mathrm{R}$ & A & $\mathrm{R}$ & A & $\mathrm{R}$ & A & $\mathrm{R}$ & A & $\mathrm{R}$ & A & $\mathrm{R}$ & A \\
\hline Drug & -8.12 & -8.25 & 0.23 & 0.64 & -3.95 & -3.81 & 4.18 & 4.45 & 1.86 & 1.63 & 3.07 & 2.94 & --- & --- \\
\hline ACE2 & -8.31 & -8.31 & 1.01 & 1.01 & -3.65 & -3.65 & 4.66 & 4.66 & 1.43 & 1.43 & 2.88 & 2.88 & --- & --- \\
\hline S PROTEIN & -7.46 & -7.46 & 1.21 & 1.21 & -3.13 & -3.13 & 4.34 & 4.35 & 1.13 & 1.13 & 3.73 & 3.73 & --- & --- \\
\hline $\begin{array}{l}\text { ACE2- S } \\
\text { PROTEIN }\end{array}$ & -7.53 & -7.53 & 1.04 & 1.04 & -3.25 & -3.25 & 4.29 & 4.29 & 1.23 & 1.23 & 3.66 & 3.66 & -41.60 & -41.60 \\
\hline ACE2-Drug & -8.08 & -7.43 & 0.26 & 0.42 & -3.91 & -3.51 & 4.17 & 3.93 & 1.83 & 1.56 & 3.11 & 3.76 & -15.61 & 1.34 \\
\hline $\begin{array}{l}\text { S PROTEIN } \\
\text { - Drug }\end{array}$ & -7.46 & -8.26 & 0.22 & 0.43 & -3.62 & -3.92 & 3.84 & 4.35 & 1.71 & 1.76 & 3.73 & 2.93 & -11.16 & 1.59 \\
\hline $\begin{array}{l}\text { ACE2- Drug- } \\
\text { S PROTEIN }\end{array}$ & -7.46 & -7.40 & 0.23 & 0.53 & -3.62 & -3.44 & 3.85 & 3.97 & 1.70 & 1.49 & 3.73 & 3.79 & -66.15 & -15.29 \\
\hline
\end{tabular}

The reactivity parameters given in Table 1 , namely the chemical potential $(\mu)$, the hardness $(\eta)$, the electrophilicity $(\omega)$, and the nucleophilicity parameter $(\mathrm{N})$ are defined in $[12,13,15,16]$. Inspection of Table 1 reveals that the calculated electrophilicity $(\omega)$ and nucleophilicity $(\mathrm{N})$ of Remdesivir is higher than that of Aspirochlorine, and consequently reflects its enhanced reactivity. Moreover, 
the chemical potential $(\mu)$ of remdesivir is higher, and its hardness $(\eta)$ is lower than that of Aspirochlorine. This provides a supportive information to conclude that remdesivir is of higher reactivity than Aspirochlorine.
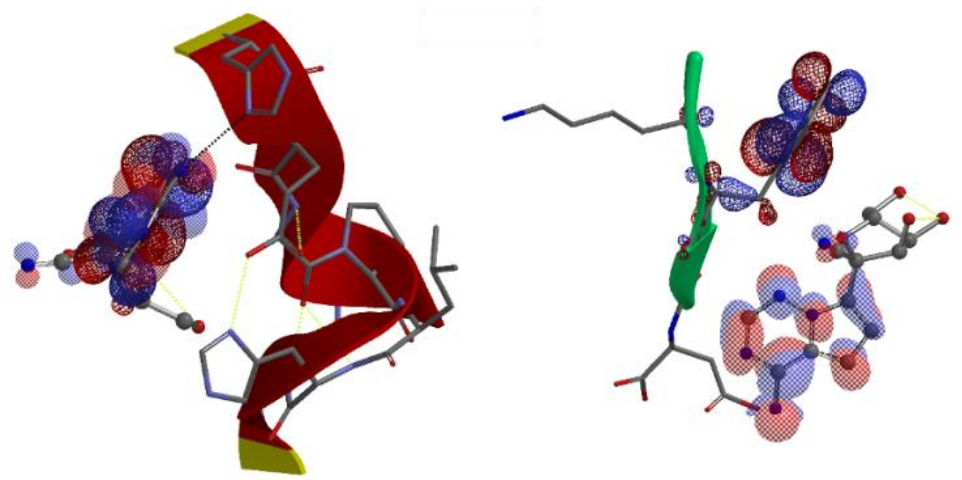

Figure 3. Left pane: Remdesivir interacts with the ACE2 via intermolecular hydrogen bond formation (black dotted line) and both HOMO (mesh) and LUMO (transparent) are localized on the drug molecule. Right pane: Remdesivir behaves like an electrophile towards the $S$ protein.

Remdesivir associates with ACE2 via intermolecular hydrogen bonding (see Figure 3). The HOMO and LUMO of the complex are localized on the drug. Both orbitals destabilized due to the exothermic complex formation with ACE2 (see Table 1). Remdesivir forms an exothermic complex with S protein, where the drug acts as an electron sink and the energy of its HOMO is ($7.46 \mathrm{eV})$ significantly destabilized relative to the free drug $(-8.12 \mathrm{eV})$, while its LUMO was almost unchanged. The same observation was noticed in the association of the drug with both ACE2 and $\mathrm{S}$ proteins. However, referring in Table 1, the presence of remdesivir results in a significant stabilization of the binding energy of the ACE2 - S protein adduct (from -41.60 to -66.15 $\mathrm{kcal} / \mathrm{mol}$ ). This extra stabilization in the binding energy enables the drug to function on the system and to shield ACE2 from the intermolecular electronic interaction with the S protein (Figure 4). 


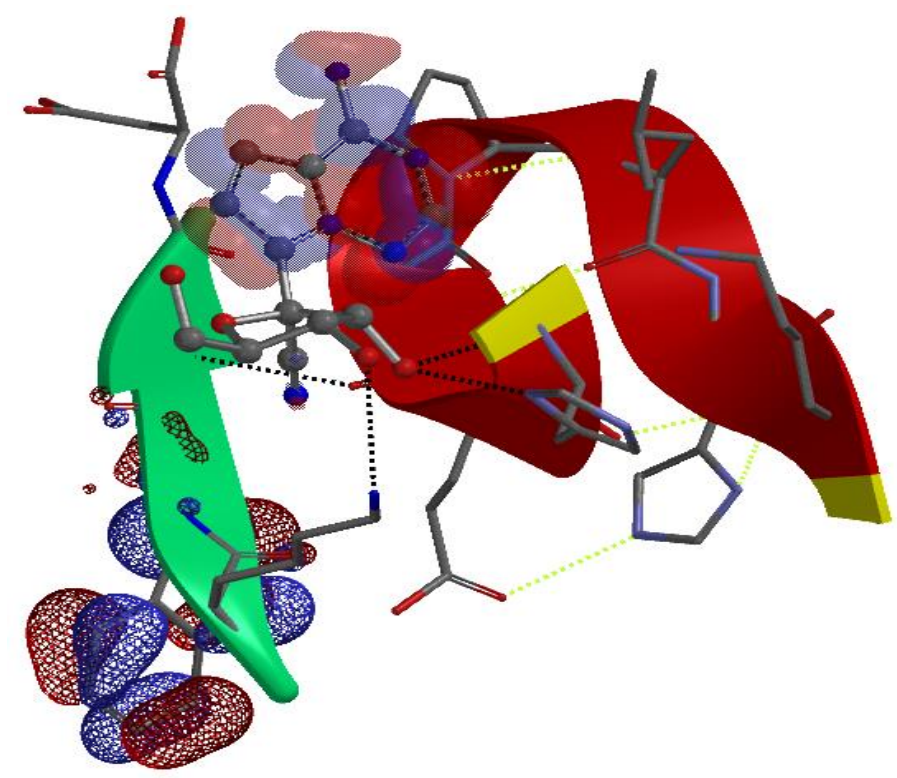

Figure 4. Remdesivir (ball and spoke model) association with ACE2 and the S protein (Tubes model). HOMO is located on the $S$ protein and the LUMO on the drug reflecting its nucleophilicity towards S protein. ACE2 is associated via intermolecular hydrogen bond formation (black dotted lines).

Aspirochlorine forms a weak complex with the ACE2 - S protein with a binding energy of $-15.29 \mathrm{k} \mathrm{cal} / \mathrm{mol}$. Moreover, the binding energy of Aspirochlorine with ACE2 or S protein was endothermic (see Table 1) pointing to an unstable adduct formation. The role could be seen by inspecting Figure 5, where it shields ACE2 from the intermolecular electronic interaction with the S protein. 


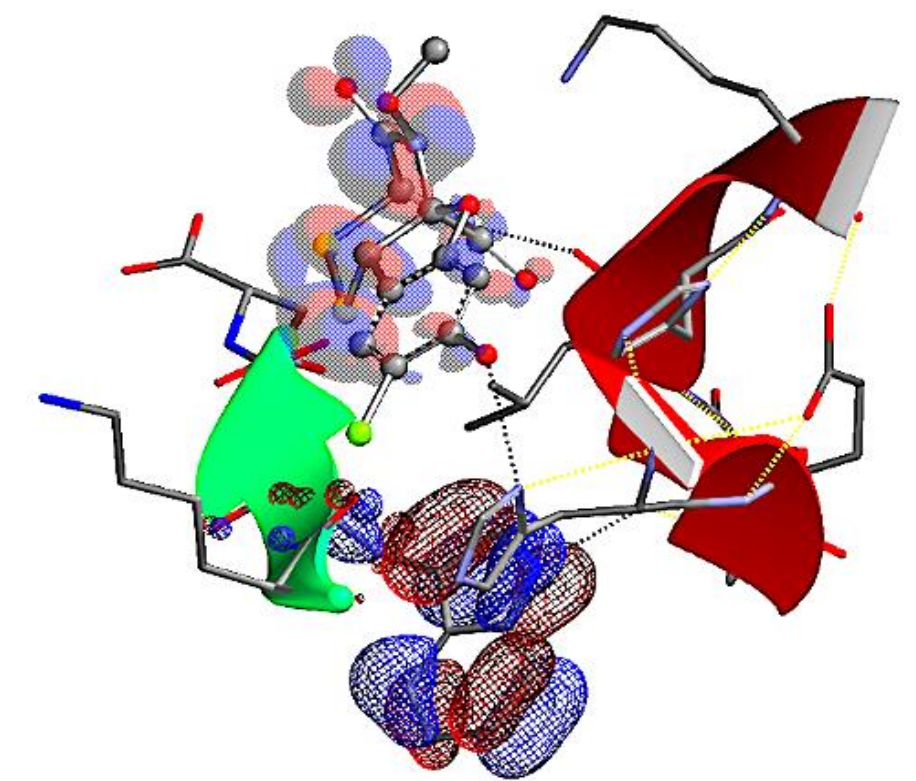

Figure 5. Aspirochlorine (ball and spoke model) association with ACE2 and the S protein (Tubes model). HOMO is located on the $S$ protein and the LUMO on the drug reflecting its nucleophilicity towards $S$ protein. ACE2 is associated via intermolecular hydrogen bond formation (black dotted lines). Remdesivir behaves similarly.

\section{4- Conclusion}

This report is associated with an ongoing coronavirus outbreak. We selected Remdesivir (used in the treatment of Ebola) and Aspirochlorine (a natural product found in Aspergillus oryzae), and their binding to specific peptide sequences of the coronavirus S-protein: ACE2 interface-drug binding adduct were calculated. The stable intermolecular adducts between the chosen drug molecules with the S protein and ACE2 result in limited host-virus interactions. The electrophilicity and nucleophilicity indices of the drugs showed that both drugs act as electron sinks to shield ACE2 from interacting with the S protein. Aspirochlorine acts as an electron acceptor (electrophile) toward both individual targets, the ACE2, and S proteins (nucleophiles). Aspirochlorine electronically shields ACE2 from the interaction with S protein by sinking the electronic charge of the $\mathrm{S}$ protein. The electrophilicity and nucleophilicity parameters of Remdesivir were higher than those of ACE2, and both molecules were bound via hydrogen bonding intermolecular interactions without intermolecular electron transfer. Remdesivir also shields ACE2 from the S protein. The results obtained strongly suggest the beneficial use of both drugs. The results reported indicate that the association of remdesivir with the target proteins was 
exothermic, while it was endothermic in the case of Aspirochlorine. Both drugs offer protection and/or treatment against the coronavirus S-protein COVID-19. Interestingly, adding the drug to the ACE2- $\mathrm{S}$ protein association is much more energetically favorable in the case of remdesivir $(\mathrm{BE}=-66.15 \mathrm{kcal} / \mathrm{mol})$ relative to the ACE2-S protein adduct $(-41.6 \mathrm{kcal} / \mathrm{mol})$ in the case of aspirochlorine $(\mathrm{BE}=-15.29 \mathrm{kcal} / \mathrm{mol})$. In other words, Remdesivir association with ACE2 $-\mathrm{S}$ protein should result in a stable complex, enabling the drug to function on the system. These findings also indicate that these simple simulation methods could rapidly help with the search for efficient drugs to get rid of COVID-19. In vivo and in vitro experimental validation of remdesivir is currently subjected to global underway testing.

\section{Conflict of interests}

There are no conflicts of interest to declare. All data are available to share upon request from the corresponding author. 


\section{References}

[1] C. Scavone, S. Brusco, M. Bertini, L. Sportiello, C. Rafaniello, A. Zoccoli A, et al. (April 2020). "Current pharmacological treatments for COVID-19: what's next?". British Journal of Pharmacology. doi:10.1111/bph.15072. PMID 32329520.

[2] "Emergency Use Authorization (EUA) of Remdesivir for Coronavirus Disease 2019

(COVID-19)". ttps://www.fda.gov/media/137566/download]

[3] "'Solidarity' clinical trial for COVID-19 treatment". World Health Organization. 27 April 2020. Retrieved 1 May 2020

[4] Yuta Tsunematsu, Naoya Maeda, Mamoru Yokoyama, Pranatchareeya Chankhamjon, Kenji Watanabe, Kirstin Scherlach, Christian Hertweck, Enzymatic Amide Tailoring Promotes RetroAldol Amino Acid Conversion to Form the Antifungal Agent Aspirochlorine, Angewandte Chemie 2018 October 22, 57 (43): 14051-14054

[5] F. Monti, F. Ripamonti, SP Hawser, K. Islam, Aspirochlorine: a highly selective and potent inhibitor of fungal protein synthesis. A natural product found in Aspergillus oryzae, ]. J Antibiot (Tokyo). 1999 Mar;52(3):311-8]

[6] Kevin M. Gayler, Kyle M. Lambert, John L. Wood, Synthetic studies towards the penicisulfuranols: Synthesis of an advanced spirocyclic diketopiperazine intermediate, Tetrahedron 75 (2019) 3154-3159

[7] M. S. A. Abdel-Mottaleb, and Yousra Abdel-Mottaleb (2020): In Search for Effective and Safe Drugs Against SARS-CoV-2: Part I] Simulated Interactions Between Selected Nutraceuticals, ACE2 Enzyme and S Protein Simple Peptide Sequences. ChemRxiv. Preprint. https://doi.org/10.26434/chemrxiv.12155235.v1

[8] M. S. A. Abdel-Mottaleb, and Yousra Abdel-Mottaleb (2020): In Search for Effective and Safe Drugs Against SARS-CoV-2: Part II] the Role of Selected Salts and Organometallics of Copper, Zinc, Selenium, and Iodine Food Supplements. ChemRxiv. Preprint. https://doi.org/10.26434/chemrxiv.12234743.v1

[9] Spartan'18, Wavefunction, Inc., Irvine, CA, USA]

[10] J.-D. Chai and M. Head-Gordon, "Long-range corrected hybrid density functionals with damped atom-atom dispersion corrections," Phys. Chem. Chem. Phys., 10 (2008) 6615-20. DOI: $10.1039 / \mathrm{B} 810189 \mathrm{~B}]$ 
[11] M. Cossi, N. Rega, G. Scalmani, V. Barone, "Energies, structures, and electronic properties of molecules in solution with the C-PCM solvation model”, J. Comput. Chem., Vol 24, no 6, pp. 669-681, 2003.

[12] Geerlings P., de Proft F., and Langenaeker W., "Conceptual density functional theory" Chemical Reviews, Vol 103, no 5, pp. 1793-1873, 2003

[13] Luis R. Domingo, Mar Ríos-Gutiérrez and Patricia Pérez, "Applications of the Conceptual Density Functional Theory Indices to Organic Chemistry Reactivity”, Molecules, Vol 21, no 748, 22 pages, 2016.

[14] Luis R. Domingo, "Molecular electron density theory: A modern view of reactivity in organic chemistry”, Molecules, Vol 21, no 1319, 15 pages, 2016.

[15] F. Zielinski, V. Tognetti and L. Joubert, "Condensed descriptors for reactivity: a methodological study", Chemical Physics Letters, Vol 527, pp. 67-72, 2012. 